\title{
Technosignatures as a Priority in Planetary Science
} A White Paper for the Planetary Science Decadal Survey 2020

\author{
Primary Author: \\ Sofia Z. Sheikh \\ Penn State University / Penn State Extraterrestrial Intelligence Center \\ szs714@psu.edu, 864-940-7093
}

Co-authors:

Anamaria Berea, George Mason University

Ross Davis, Indiana University

Gabriel G. De la Torre, University of Cadiz. Spain

Julia DeMarines, University of California, Berkeley

Theresa Fisher, School of Earth and Space Exploration, Arizona State University

Searra Foote, Arizona State University

Dawn Gelino, Caltech / IPAC-NASA Exoplanet Science Institute

David Grinspoon, Planetary Science Institute, Tucson

George Profitiliotis, National Technical University of Athens, Greece

Alexander M. Sessa, Georgia Institute of Technology

Caitlyn Singam, University of Maryland, College Park

Evan L. Sneed, Penn State University / Penn State Extraterrestrial Intelligence Center

Clément Vidal, Center Leo Apostel, Vrije Universiteit Brussels, Belgium

Jason T. Wright, Penn State University / Penn State Extraterrestrial Intelligence Center

130 Additional Signatories listed at this link.

\begin{abstract}
:
Are we alone? The study of technosignatures, physical manifestations of technology from extraterrestrial intelligences, is a rigorous astrobiological sub-discipline. The Planetary Science decadal should include searches for technosignatures alongside biosignatures in its recommendations for funding a robust astrobiology research portfolio.
\end{abstract}




\section{Technosignatures belong under the umbrella of planetary science}

A technosignature is "any physical manifestation of technology" [1], or, in the framework usually applied to biosignatures, "an object, substance, and/or pattern whose origin specifically requires a [technological] agent." [2] The discovery of a technosignature could be the first detection of life beyond Earth, and provide incredible insight into the evolution of life in the universe.

The search for technosignatures is generally considered under the umbrella of the Search for Extraterrestrial Intelligence, or SETI. However, in the enabling context of the exoplanet revolution, and with the ability to perform spectroscopic characterization of planetary systems using the assets of upcoming space telescope missions, the search for proposed technosignatures has expanded to a broader range beyond traditional radio searches. The number of possible technosignatures has increased greatly over the past decade, much like the explosion of proposed biosignatures (including, e.g., the immense number of potential biosignature gases [3]). Not only are proposed biosignatures and proposed technosignatures both proliferating in the literature, their approaches are also converging. For example, when we perform near-future spectroscopic searches for atmospheric biosignatures such as oxygen, we can, with little effort, obtain the necessary data for a search for atmospheric technosignatures such as $\mathrm{NO}_{\mathrm{x}}$ gases or chlorofluorocarbons.

Indeed, technosignatures may be more obvious, more ubiquitous, and more unambiguous than biosignatures in the Solar System and in the Galaxy. Technology can drive changes at a level similar to or greater than that of a planet's biosphere [4]. Since technological life can, in principle, spread among the stars, these markers of technology may be found more often than habitable exoplanets. [5] pointed out that despite the long travel times, the efficiency of and capacity for exploration and communication is much larger for interstellar probes than for interstellar remote sensing or transmission, and so the even the Solar System may be home to technology. While this idea has lost much of its academic support, especially with the advent of interplanetary probes and planetary surface maps that show no obvious signs of technology, quantified upper limits have yet to be placed.

With this context, the search for technosignatures is clearly part of the spectrum of astrobiology and a crucial consideration for planetary science. Almost all of the science themes listed in the Planetary Science Decadal White Paper call relate to technosignatures in some way. Technosignature searches have been proposed for every terrestrial and primitive body within the Solar System. There is a plethora of proposed technosignatures for exoplanets in the era of the James Webb Space Telescope and beyond. Furthermore, theoretical technosignature work falls squarely in the astrobiological realm of determining the origin, likelihood, and distribution of life in the universe.

Scientifically, technosignatures are just another facet of astrobiology. But technosignature researchers, under the banner of SETI, have often been siloed away from the astrobiological umbrella. If, instead, we can integrate technosignature searches into the general planetary science community, we will encourage a collaboration and exchange of ideas which will benefit practitioners of both disciplines. The Planetary Science decadal should include searches for technosignatures alongside biosignatures in its recommendations for funding a robust astrobiology research portfolio. 


\section{Technosignatures align with agency priorities}

Technosignature research complements all aspects of the NASA 2020-2024 Science Mission Plan. At the largest scales, the stated mission of the NASA Science Mission Directorate is to: "Discover the secrets of the universe. Search for life elsewhere. Protect and improve life on Earth." [6] Technosignature searches are searches for life elsewhere that seek to answer the questions "where did we come from?" and "are we alone?". Just like traditional astrobiology searches, technosignature studies provide an excellent opportunity for cross-divisional collaboration within NASA. To move the field forward, we must make advances in planetary sciences (e.g., the development of ground-penetrating radar for lunar applications), in astrophysics (e.g., better understanding stellar activity to obtain higher-precision stellar photometry for transit missions), in Earth science (e.g., developing ChipSat technology for climate measurements and remote sensing) and in heliophysics (e.g., laying the groundwork for heliocentric "static satellites" that could be used for in situ investigations of interstellar comets such as 'Oumuamua).

Within the Planetary Science division, NASA's goal is to better understand our solar system. Searches for technosignatures within the solar system include free-floating artifacts (perhaps from an extraterrestrial intelligence's "Breakthrough Starshot" [7] program), or traces of technological activity on another terrestrial solar system body. By completing such searches, we not only look for traces of life, but we would also learn more about the population and distribution of small bodies in the solar system, improve our characterization of surface imagery from NASA missions such as Dawn and the Lunar Reconnaissance Orbiter ${ }^{1}$, and develop machine learning methods to find interesting natural anomalies within the solar system. Modeling work relevant to solar system technosignatures, such as investigating the residence times of artificial satellites in particular solar system orbits, or calculating the survival time of artificial structures on different terrestrial bodies, will help us better understand the hazards to human technology that will be deployed in the course of human space exploration. These strategies also have direct relevance to planetary defense: we automatically develop a better understanding of the local space environment in the normal course of performing multi-band visual observations and radar shape characterizations searching for artificial (non-human) near-Earth objects.

Additionally, technosignatures are uniquely positioned to create opportunities for scientific discovery at the interface between planetary sciences and the other divisions, especially Earth Sciences. The Anthropocene, a proposed epoch to describe present-day Earth, is characterized by human technosignatures on the planet's surface and involves forces that may possibly dominate the future of our planet's habitability and alter our entire biosphere. We can use our understanding of this technology's effect on the Earth, including symptoms such as atmospheric pollution, ecosystem change, sea-level rise, and climate volatility, to search for and model technology's effect on other planets and vice versa.

Broadly, technosignature studies can be divided into "hypothesis-driven" model-based searches and "serendipity-driven" anomaly searches. Hypothesis-driven searches begin with theorized technosignatures based on perceived universalities (e.g., the usefulness of electromagnetic waves for communication, or the need for energy harvesting to sustain

\footnotetext{
${ }^{1}$ Both of which are already being used in technosignature research e.g., [8][9]
} 
large-scale technological activity) and are tested with observational campaigns. Serendipity-driven searches begin with large datasets, and use anomaly detection methods to search for objects within those sets whose properties imply a different classification or generation mechanism ${ }^{2}$. Technosignature searches are not explicitly encouraged in the research portfolios of most current funding organizations, partially because of a perception that the science is "high-risk," or high resource-expenditure with low science-return. But neither of these classes of technosignature study is high-risk. Hypothesis-driven searches set upper limits on the existence of technosignatures if no technosignatures are discovered, quantifying the distribution of technology in the universe. Serendipity-driven searches have a high return of ancillary scientific benefits - for example, the discovery of instrumental artifacts and astrophysical outliers. Thus, so long as we deconvolve a study's perceived riskiness from its guarantee of a technosignature discovery, these studies are much lower in risk than they are traditionally treated. NASA even encourages "a willingness to learn and change and to take risks in the interests of science" in high-impact studies, tempered by the priorities of the scientific community.

The partnerships summarized in Section 5.3.3 of the 2018 NASA Technosignatures Workshop report [10], promote not just cross-disciplinary but also inter-agency opportunities. NOAA has near-Earth space assets that could be leveraged, for example, in the search for near-Earth technosignatures.

Finally, to truly give back to the community, a strong science program must be associated with a strong outreach and communication program. NASA wants to engage with learners of all ages to share its scientific story and mission, and few topics ignite the interest of the public like technosignatures. The technosignature community would be delighted to collaborate with outreach venues such as the National Air and Space Museum to design interactive and interdisciplinary exhibits where the public could learn more about historic and ongoing technosignature searches at NASA.

\section{Technosignatures will strengthen other astrobiological research priorities}

Serendipity-driven technosignature searches involve re-analyzing large repositories of data from astronomy with newer methods in data science such as machine learning and deep learning. Some data science and machine learning applications for technosignature searches involve simulating data for planetary atmospheres (in the context of astrobiology), simulating and analyzing exoplanet data from both the Kepler and TESS missions, as well as understanding limits from information theory or the discovery of universal laws of information. For example, NASA Frontier Development Lab (FDL) had two challenges in 2018 related to artificial intelligence and deep learning techniques for the discovery of exoplanetary transits and for simulations of exoplanetary atmospheres ${ }^{3}$ [11]. At FDL 2020, one of the challenges involved the development of a reverse image search engine for Earth Observation (EO) unlabeled data, and another challenge dealt with the discovery of star-spots using image processing techniques from

\footnotetext{
${ }^{2}$ As explored in the KISS Technosignatures Workshop 2019: https://kiss.caltech.edu/workshops/technosignatures/technosignatures.html

${ }^{3}$ Exo-ATMOS, an open-source Python package for simulating exoplanetary atmospheres, was developed at FDL
} 
the Kepler mission. All of these challenges have direct applications to both technosignature and non-technosignature based research within the realm of big data.

Breakthrough Listen (BL) has been particularly invested in data science and technosignature research efforts, conducting some of the most comprehensive searches to date [12]. Their new methods are rapidly developing alongside advancements in the computer science and data science fields, presenting new opportunities for searches in both old and new data that can be easily used for the analysis of planetary data [13]. Some of these methods include unsupervised learning, Bayesian inferences, neural networks, and many more [13, 14]. Vice-versa, new computational algorithms and packages are being developed due to technosignature research; for example, the ORBITS observation manager, at the Green Bank Observatory, was developed through the BL program. Other software and tutorials have been released via the BL group to analyze radio data in .hdf5/.h5 files and perform RFI excision. These tools are available now to researchers and the public-at-large alongside many open data repositories that include results from Voyager to Kepler and TESS.

The search for technosignatures is also relevant to astrobiology in the context of Earth's entry into an "Anthropocene Epoch" [15]. Indeed, one way to define the Anthropocene is the moment in geological time when Earth first developed technosignatures [16]. Astrobiology is in large part a study of the relationship between life and planets, and on Earth the Anthropocene represents a new phase in that relationship in which cognitive/technical processes have become planetary scale processes [17]. Pragmatically, anthropogenic changes alter the "baseline" from which we study comparative planetology, and since, implicitly or explicitly, Earth forms the standard of comparison by which we measure other planetary histories and processes, it is important to be aware that our observations are made from an altered and rapidly changing platform. The Anthropocene arguably marks a branching point in planetary history, one which may have analogs elsewhere in the galaxy, and thus may determine what we search for on some exoplanets and how we interpret our observations. One way of framing the potential for long term survival of human technological civilization on Earth is whether the changes wrought by the "Early Anthropocene" which we now find ourselves in, characterized by inadvertent planetary-scale changes caused by powerful, planet-changing technologies deployed without any long-term global plan, can transition into a "Mature Anthropocene" characterized by feedbacks between awareness of our planetary role and more thoughtful deployment of technologies, allowing sustainable long-term integration of human activities into global cycles. Framed this way, the choices facing humanity in the $21^{\text {st }}$ century resonate with a central question of technosignature searches: "Is it possible for a global technological civilization to form a stable, long-term relationship with an evolving planet"? [16] Overall, the study of technosignatures is closely related to current physical and social science findings involving global warming and other environmental challenges facing humanity.

Finally, technosignature research has a possibility of enhancing work in the social sciences as well as the physical sciences. Most directly, collaboration with the social sciences could allow us to decode and interpret information from potential technosignatures and contribute to better understanding of the findings [18]. In addition, social science studies can assist us with analyzing our methods and interpretations of data and in minimizing bias in both human and machine-learning mediated strategies.

Technosignature research is an integral part of the diverse field of astrobiology. Not only is it philosophically in continuation with searches for biosignatures (and even more scientifically 
promising along certain axes), but it also synergizes with multiple NASA priorities for the next decade and beyond. Technosignature searches, given the opportunity to find a home in the planetary science community, will drive innovations beyond their own sub-field. Within this new home, we hope to share advances in machine learning, big data, and image processing along with a deeper understanding of life, both from our own planet and beyond.

\section{References}

[1] Wright, Jason T., et al. "Recommendations from the ad hoc committee on SETI nomenclature." arXiv preprint arXiv:1809.06857 (2018).

[2] Des Marais, David J., et al. "The NASA astrobiology roadmap." Astrobiology 8.4 (2008): 715-730.

[3] Seager, Sara, W. Bains, and J. J. Petkowski. "Toward a list of molecules as potential biosignature gases for the search for life on exoplanets and applications to terrestrial biochemistry." Astrobiology 16.6 (2016): 465-485.

[4] Frank, Adam, Axel Kleidon, and Marina Alberti. "Earth as a hybrid planet: the Anthropocene in an evolutionary astrobiological context." Anthropocene 19 (2017): 13-21.

[5] Bracewell, Ronald N. "Communications from superior galactic communities." Nature 186.4726 (1960): 670-671.

[6] NASA Science Mission Directorate (2020). Science 2020-2024: A Vision for Scientific Excellence. https://science.nasa.gov/about-us/science-strategy

[7] Parkin, Kevin LG. "The breakthrough starshot system model." Acta Astronautica 152 (2018): 370-384.

[8] De la Torre,G. G. "Does artificial intelligence dream of non-terrestrial technosignatures?." Acta Astronautica 167 (2020): 280-285.

[9] Lesnikowski, Adam, Valentin T. Bickel, and Daniel Angerhausen. "Unsupervised Distribution Learning for Lunar Surface Anomaly Detection." arXiv preprint arXiv:2001.04634 (2020).

[10] NASA Technosignatures Workshop Participants (2018). NASA and the Search for Technosignatures: A Report from the NASA Technosignatures Workshop. https://arxiv.org/abs/1812.08681

[11] https://frontierdevelopmentlab.org

[12] https://breakthroughinitiatives.org/news/30, retrieved September 10, 2020. 
[13] Berea, A., Croft, S., \& Angerhausen, D. (2019). The Promise of Data Science for the Technosignatures Field. arXiv preprint arXiv:1903.08381.

[14] Haqq-Misra, J., Berea, A., Balbi, A., \& Grimaldi, C. (2019). Searching for Technosignatures: Implications of Detection and Non-Detection. arXiv preprint arXiv:1903.06550.

[15] Steffen W., Crutzen P. J.\& McNeill J. R.. 2007 The Anthropocene: are humans now overwhelming the great forces of Nature. Ambio 36, 614-621.

[16] Grinspoon, D. (2020). Cognitive Planetary Transitions: Is there a "Sapiezoic Eon" in Earth's future or elsewhere in the galaxy?, Invited Keynote talk at the TechnoClimes Workshop, August 5, 2020, https://www.youtube.com/watch?v=Db7nCDC W9g

[17] Grinspoon, David. 2016. Earth in Human Hands: Shaping Our Planet's Future. Grand Central Publishing.

[18] Harrison, A., Billingham, J., Dick, S. J., Finney, B., Michaud, M. A., Tarter, D. E., ... \& Vakoch, D. A. (2000). The role of the social sciences in SETI. Tough (2000b), section V, paper, 1, 71-85. 